\title{
On common approximate fixed points of monotone nonexpansive semigroups in Banach spaces
}

\author{
Mostafa Bachar ${ }^{1 *}$ and Mohamed A Khamsi2,3
}

\section{"Correspondence:}

mbachar@ksu.edu.sa

'Department of Mathematics, College of Sciences, King Saud University, Riyadh, Saudi Arabia Full list of author information is

available at the end of the article

\section{每 Springer}

\begin{abstract}
In this paper, we investigate the common approximate fixed points of monotone nonexpansive semigroups of nonlinear mappings $\{T(t)\}_{t \geq 0}$, i.e., a family such that $T(0) x=x, T(s+t) x=T(s) \circ T(t) x$, where the domain is a Banach space. In particular we prove that under suitable conditions, the common approximate fixed points are the same as the common approximate fixed points set of two mappings from the family. Then we give an algorithm of how to construct an approximate fixed point sequence of the semigroup in the case of a uniformly convex Banach space.
\end{abstract}

MSC: Primary 47H09; secondary 46B20; 47H10; 47E10

Keywords: approximate fixed point; common approximate fixed point; fixed point; common fixed point; integral equation; monotone nonexpansive mapping; semigroup

\section{Introduction}

Nonexpansive mappings are those maps which have Lipschitz constant equal to 1 . The fixed point theory for such mappings is rich and varied. It finds many applications in nonlinear functional analysis. The existence of fixed points for nonexpansive mappings in Banach and metric spaces has been investigated since the early 1960s; see, e.g., Belluce and Kirk [1, 2], Browder [3], Bruck [4], Lim [5].

In recent years, a new direction has been very active essentially after the publication of Ran and Reurings fixed point theorem [6] dealing with the extension of the Banach contraction principle to metric spaces endowed with a partial order. In particular, they show how this extension is useful when dealing with some special matrix equations. It is worth mentioning that similar results were discovered by Turinici $[7,8]$. Another similar approach was carried out by Nieto and Rodríguez-López [9] and used such arguments in solving some differential equations. In [10] Jachymski gave a more general unified version of these extensions by considering graphs instead of a partial order.

The purpose of this paper is to prove the existence of approximate fixed points for semigroups of nonlinear monotone mappings acting in a Banach vector space endowed with a partial order. Note that from a numerical point of view, approximate fixed points are very useful since exact fixed points may be hard to find. Moreover, we will also give an algorithm of how to build such approximate fixed points. Let us recall that a family $\{T(t)\}_{t \geq 0}$ of

(c) 2015 Bachar and Khamsi. This article is distributed under the terms of the Creative Commons Attribution 4.0 International License (http://creativecommons.org/licenses/by/4.0/), which permits unrestricted use, distribution, and reproduction in any medium, provided you give appropriate credit to the original author(s) and the source, provide a link to the Creative Commons license, and indicate if changes were made. 
mappings forms a semigroup if $T(0) x=x$ and $T(s+t) x=T(s) T(t)$. Such a situation is quite typical in mathematics and applications. For instance, in the theory of dynamical systems, the vector function space would define the state space and the mapping $(t, x) \rightarrow T(t) x$ would represent the evolution function of a dynamical system. In the setting of this paper, the state space is a Banach space. Our approach is new and different from the ideas found in $[6-9,11]$. Indeed when one deals with a contraction, the focus is usually on the distance being complete. But when dealing with nonexpansive mappings, then geometric properties of the space are crucial.

For more on metric fixed point theory, the reader may consult the books [12,13]. As for the semigroup theory, we suggest the references [14-16].

\section{Preliminaries}

Let $(X,\|\cdot\|)$ be a Banach vector space and suppose that $\preceq$ is a partial order on $X$. Throughout, we will assume that the partial order $\preceq$ and the liner structure of $X$ enjoys the following convexity property:

$$
a \preceq b \text { and } c \preceq d \quad \Longrightarrow \quad \alpha a+(1-\alpha) c \preceq \alpha b+(1-\alpha) d
$$

for any $\alpha \in[0,1]$ and $a, b, c, d \in X$. Moreover, we will assume that order intervals are closed. Recall that an order interval is any of the subsets

(i) $[a, \rightarrow)=\{x \in X ; a \preceq x\}$,

(ii) $(\leftarrow, a]=\{x \in X ; x \preceq a\}$,

for any $a \in X$. Next we give the definition of monotone nonexpansive mappings.

Definition 2.1 Let $(X,\|\cdot\|, \preceq)$ be as above. Let $C$ be a nonempty subset of $X$. A map $T: B \rightarrow X$ is said to be

(a) monotone if $T(x) \preceq T(y)$ whenever $x \preceq y$;

(b) monotone nonexpansive if $T$ is monotone and

$$
\|T(x)-T(y)\| \leq\|x-y\|
$$

for any $x, y \in C$ such that $x \preceq y$.

A fixed point of $T$ is any element $x \in C$ such that $T(x)=x$. The set of all fixed points of $T$ is denoted by $\operatorname{Fix}(T)$. A sequence $\left\{x_{n}\right\}$ is called an approximate fixed point sequence of $T$ if $\lim _{n \rightarrow \infty}\left\|T\left(x_{n}\right)-x_{n}\right\|=0$. The set of approximate fixed point sequences of $T$ will be denoted by $\operatorname{AFPS}(T)$.

This definition is extended to the case of semigroup of mappings.

Definition 2.2 Let $(X,\|\cdot\|, \preceq)$ be as above. Let $C$ be a nonempty subset of $X$. A oneparameter family $\mathcal{F}=\{T(t) ; t \geq 0\}$ of mappings from $C$ into $C$ is said to be a monotone nonexpansive semigroup if $\mathcal{F}$ satisfies the following conditions:

(i) $T(0) x=x$ for $x \in C$;

(ii) $T(t+s)=T(t) \circ T(s)$ for $t, s \in[0, \infty)$;

(iii) for each $t \geq 0, T(t)$ is a monotone nonexpansive mapping. 
Define then the set of all common fixed points of $\mathcal{F}$ as $\operatorname{Fix}(\mathcal{F})=\bigcap_{t \geq 0} \operatorname{Fix}(T(t))$. Similarly, define the set of approximate point sequences of $\mathcal{F}$, denoted by $\operatorname{AFPS}(\mathcal{F})$, as $\operatorname{AFPS}(\mathcal{F})=$ $\bigcap_{t \geq 0} \operatorname{AFPS}(T(t))$.

Next we give an example of such semigroup.

Example 2.1 Let $(X,\|\cdot\|, \preceq)$ be as above. Let $C$ be a nonempty closed, bounded convex subset of $X$. Let $J: C \rightarrow C$ be a monotone nonexpansive mapping. Let $x \in C$ be such that $x \preceq J(x)$. Consider the recurrent sequence defined by

$$
\left\{\begin{array}{l}
u_{0}(t)=x \\
u_{n+1}(t)=e^{-t} x+\int_{0}^{t} e^{s-t} J\left(u_{n}(s)\right) d s
\end{array}\right.
$$

for any $t \in[0, A]$, where $A$ is a fixed positive number. Then the sequence $\left\{u_{n}(t)\right\}$ is Cauchy for any $t \in[0, A]$. Indeed, let us suppose that $x, y:[0, A] \rightarrow X$ are continuous functions. For each $t \in[0, A]$, we have

$$
\left\|e^{-t} y(t)+\int_{0}^{t} e^{s-t} x(s) d s\right\| \leq e^{-t}\|y\|_{\infty}+K(t)\|x\|_{\infty}
$$

where $\|u\|_{\infty}=\sup \{\|u(s)\| ; t \in[0, A]\}$ and $K(t)=\int_{0}^{t} e^{s-t} d s=1-e^{-t}$. Indeed, let $t \in[0, A]$ be fixed, and $\tau=\left\{t_{i} ; i=0,1, \ldots, n\right\}$ be any subdivision of $[0, t]$. Set

$$
S_{\tau}=e^{-t} y(t)+\sum_{i=0}^{n-1}\left(t_{i+1}-t_{i}\right) e^{t_{i}-t} x\left(t_{i}\right) .
$$

The family $\left\{S_{\tau}\right\}$ is norm-convergent to

$$
S=e^{-t} y(t)+\int_{0}^{t} e^{s-t} x(s) d s
$$

when $|\tau|=\sup \left\{\left|t_{i+1}-t_{i}\right| ; i=0,1, \ldots,(n-1)\right\}$ goes to 0 . Since

$$
\sup _{s \in[0, t]}\|x(s)\| \leq \sup _{s \in[0, A]}\|x(s)\|=\|x\|_{\infty}
$$

and

$$
\left\|S_{\tau}\right\| \leq e^{-t}\|y(t)\|+\sum_{i=0}^{n-1}\left(t_{i+1}-t_{i}\right) e^{t_{i}-t}\|x\|_{\infty} \leq e^{-t}\|y(t)\|+K(t)\|x\|_{\infty},
$$

where we used

$$
\sum_{i=0}^{n-1}\left(t_{i+1}-t_{i}\right) e^{t_{i}-t} \leq \int_{0}^{t} e^{s-t} d s=K(t)
$$

then we have $\|S\| \leq e^{-t}\|y(t)\|+K(t)\|x\|_{\infty}$. Therefore we have

$$
\left\|e^{-t} y(t)+\int_{0}^{t} e^{s-t} x(s) d s\right\| \leq e^{-t}\|y\|_{\infty}+K(t)\|x\|_{\infty}
$$


for any $t \in[0, A]$. Next we discuss the sequence $\left\{u_{n}(t)\right\}$. Since

$$
u_{1}(t)=e^{-t} x+\int_{0}^{t} e^{s-t} J(x) d s=e^{-t} x+\left(1-e^{-t}\right) J(x), \quad t \in[0, A],
$$

we have $u_{0}(t) \preceq u_{1}(t)$ for any $t \in[0, A]$. Assume that $u_{n}(t) \preceq u_{n+1}(t)$ for any $t \in[0, A]$. Then we have $J\left(u_{n}(t)\right) \preceq J\left(u_{n+1}(t)\right)$ for any $t \in[0, A]$ because $J$ is monotone. Using the properties of the partial order $\preceq$, we get

$$
u_{n+1}(t)=e^{-t} x+\int_{0}^{t} e^{s-t} J\left(u_{n}(s)\right) d s \preceq e^{-t} x+\int_{0}^{t} e^{s-t} J\left(u_{n+1}(s)\right) d s=u_{n+2}(t)
$$

for any $t \in[0, A]$. By induction, we conclude that $\left\{u_{n}(t)\right\}$ is monotone increasing for any $t \in[0, A]$. Moreover, we have

$$
u_{n+2}(t)-u_{n+1}(t)=\int_{0}^{t} e^{s-t}\left(J\left(u_{n+1}(s)\right)-J\left(u_{n}(s)\right)\right) d s, \quad t \in[0, A],
$$

which implies by using the monotone nonexpansive behavior of $J$

$$
\left\|u_{n+2}(t)-u_{n+1}(t)\right\| \leq \int_{0}^{t} e^{s-t}\left\|J\left(u_{n+1}(s)\right)-J\left(u_{n}(s)\right)\right\| d s, \quad t \in[0, A],
$$

or

$$
\left\|u_{n+2}(t)-u_{n+1}(t)\right\| \leq \int_{0}^{t} e^{s-t}\left\|u_{n+1}(s)-u_{n}(s)\right\| d s, \quad t \in[0, A]
$$

for any $n \geq 0$. From this inequality and by induction, we get

$$
\left\|u_{n+1}(t)-u_{n}(t)\right\| \leq\left(1-e^{-A}\right)^{n} \operatorname{diam}(C), \quad t \in[0, A],
$$

where $\operatorname{diam}(C)=\sup \{\|y-z\| ; y, z \in C\}<\infty$, since $C$ is bounded. Hence

$$
\left\|u_{n+h}(t)-u_{n}(t)\right\| \leq \frac{\left(1-e^{-A}\right)^{n}}{1-\left(1-e^{-A}\right)} \operatorname{diam}(C)=e^{A}\left(1-e^{-A}\right)^{n} \operatorname{diam}(C)
$$

for any $t \in[0, A]$ and $n, h \in \mathbb{N}$. Clearly this implies that $\left\{u_{n}(t)\right\}$ is Cauchy for any $t \in[0, A]$. Since order intervals are closed, we conclude that the limit $u(t)$ of $\left\{u_{n}(t)\right\}$ satisfies $u_{n}(t) \preceq$ $u(t)$ for any $n \geq 0$ and $t \in[0, A]$. Using the monotone nonexpansive behavior of $J$, we get

$$
\left\|J\left(u_{n}(t)\right)-J(u(t))\right\| \leq\left\|u_{n}(t)-u(t)\right\| \leq e^{A}\left(1-e^{-A}\right)^{n} \operatorname{diam}(C)
$$

for any $t \in[0, A]$ and $n, h \in \mathbb{N}$. In particular, we have $\lim _{n \rightarrow+\infty} J\left(u_{n}(t)\right)=J(u(t))$, uniformly in $[0, A]$, which implies

$$
u(t)=e^{-t} x+\int_{0}^{t} e^{s-t} J(u(s)) d s, \quad t \in[0, A] .
$$

Moreover, the same proof as above will show that if $x \preceq y$, then $u_{n}(t) \preceq U_{n}(t)$, for any $n, t \geq$ 0 , where $u_{n}$ and $U_{n}$ are the functions obtained by the initial values $x$ and $y$ respectively by (2.1). This will imply that $u(t) \preceq U(t)$ for any $t \geq 0$. Define the one-parameter family 


$$
\begin{gathered}
\mathcal{F}:[0, \infty) \times C \rightarrow C \text { by } \\
T(t) x=u(t) .
\end{gathered}
$$

Then $\mathcal{F}$ defines a semigroup which is monotone nonexpansive. Indeed, we have $\| u_{0}(t)-$ $U_{0}(t)\|=\| x-y\|=\| x-y \|_{\infty}$. Assume $\left\|u_{n}(t)-U_{n}(t)\right\| \leq\|x-y\|$, then by using (2.2) we will have

$$
\left\|u_{n+1}(t)-U_{n+1}(t)\right\| \leq e^{-t}\|x-y\|+\int_{0}^{t} e^{s-t}\left\|u_{n}(s)-U_{n}(s)\right\| d s \leq\|x-y\| .
$$

By induction, $\left\|u_{n}(t)-U_{n}(t)\right\| \leq\|x-y\|$ holds for any $n \in \mathbb{N}$ and $t \in[0, A]$. Hence

$$
\|T(t) x-T(t) y\|=\|u(t)-U(t)\| \leq\|x-y\|
$$

for any $t \in[0, A]$. Moreover, we have

$$
\begin{aligned}
T(t+s) x & =e^{-(t+s)} x+\int_{0}^{t+s} e^{-(t+s-\sigma)} J(T(\sigma) x) d \sigma \\
& =e^{-(t+s)} x+\int_{0}^{t} e^{-(t+s-\sigma)} J(T(\sigma) x) d \sigma+\int_{t}^{t+s} e^{-(t+s-\sigma)} J(T(\sigma) x) d \sigma \\
& =e^{-s}\left(e^{-t} x+\int_{0}^{t} e^{-(t-\sigma)} J(T(\sigma) x) d \sigma\right)+\int_{0}^{s} e^{-(s-\sigma)} J(T(t+\sigma) x) d \sigma \\
& =e^{-s} T(t) x+\int_{0}^{s} e^{-(s-\sigma)} J(T(t+\sigma) x) d \sigma \\
& =T(s)(T(t) x)
\end{aligned}
$$

for any $t \geq 0$. Note that if we started by a point $x \in C$ such that $J(x) \preceq x$, then we would have found that the sequence $\left\{u_{n}(t)\right\}$ is monotone decreasing for any $t \geq 0$. In other words, the conclusion would have been the same. Moreover, if $x$ is a fixed point of $J$, then we have $u_{n}(t)=x$ for any $n \in \mathbb{N}$ and $t \geq 0$. Hence $T(t) x=x$ for any $t \geq 0$, i.e., $x \in \bigcap_{t \geq 0} \operatorname{Fix}(T(t))$. On the other hand, it is easy to show that if $x \in \operatorname{Fix}(\mathcal{F})$, then $x \in \operatorname{Fix}(J)$, which means we have

$$
\operatorname{Fix}(J)=\bigcap_{t \geq 0} \operatorname{Fix}(T(t))
$$

\section{Common approximate fixed points of semigroups}

Before we state our first result, we need the following definition.

Definition 3.1 Let $(X,\|\cdot\|)$ be a Banach space and $C \subset X$ be nonempty. A one-parameter family $\mathcal{F}=\{T(t) ; t \geq 0\}$ of mappings from $C$ into $X$ is said to be:

(i) continuous on $C$ if for any $x \in C$, the mapping $t \rightarrow T(t) x$ is continuous, i.e., for any $t_{0} \geq 0$, we have

$$
\lim _{t \rightarrow t_{0}}\left\|T(t) x-T\left(t_{0}\right) x\right\|=0
$$

for any $x \in C$; 
(ii) strongly continuous on $C$ if for any bounded nonempty subset $K \subset C$, we have

$$
\lim _{t \rightarrow t_{0}} \sup _{x \in K}\left(\left\|T(t) x-T\left(t_{0}\right) x\right\|\right)=0
$$

The following technical lemmas will be useful throughout.

Lemma 3.1 Let $(X,\|\cdot\|)$ be a Banach space and $C \subset X$ be nonempty. Let $J: C \rightarrow C$ be a uniformly continuous mapping. Then we have $\operatorname{AFPS}(J) \subset \operatorname{AFPS}\left(J^{m}\right)$ for any $m \geq 2$.

Proof Without loss of generality we may assume that AFPS $(J)$ is not empty. Let $\left\{x_{n}\right\} \in$ $\operatorname{AFPS}(J)$, i.e., $\lim _{n \rightarrow \infty}\left\|J\left(x_{n}\right)-x_{n}\right\|=0$. Fix $m \geq 2$, then we have

$$
\left\|J^{m}\left(x_{n}\right)-x_{n}\right\| \leq \sum_{k=0}^{m-1}\left\|J^{k+1}\left(x_{n}\right)-J^{k}\left(x_{n}\right)\right\|
$$

for any $n \geq 1$. Since $T$ is uniformly continuous, then we must have

$$
\lim _{n \rightarrow \infty}\left\|J^{k+1}\left(x_{n}\right)-J^{k}\left(x_{n}\right)\right\|=0
$$

for any $k \geq 1$. Since $m$ is fixed, we get $\lim _{n \rightarrow \infty}\left\|J^{m}\left(x_{n}\right)-x_{n}\right\|=0$, i.e., $\left\{x_{n}\right\} \in \operatorname{AFPS}\left(J^{m}\right)$.

Lemma 3.2 Let $(X,\|\cdot\|)$ be a Banach space and $C \subset X$ be nonempty. Let $\mathcal{F}=\{T(t) ; t \geq 0\}$ be a one-parameter semigroup of uniformly continuous mappings from $C$ into $C$. Let $\alpha$ and $\beta$ be two positive real numbers. Then we have

$$
\operatorname{AFPS}(T(\alpha)) \cap \operatorname{AFPS}(T(\beta)) \subset \bigcap_{t \in G_{+}(\alpha, \beta)} \operatorname{AFPS}(T(t))
$$

where $G_{+}(\alpha, \beta)=\{m \alpha+k \beta \geq 0 ; m, k \in \mathbb{Z}\}$.

Proof Without loss of generality we may assume that $\operatorname{AFPS}(T(\alpha)) \cap \operatorname{AFPS}(T(\beta))$ is not empty. Let $\left\{x_{n}\right\} \in \operatorname{AFPS}(T(\alpha)) \cap \operatorname{AFPS}(T(\beta))$. Let $t \in G_{+}(\alpha, \beta)$. Then we have two cases. First assume $t=m \alpha+k \beta$, where $m, k \geq 0$. Then

$$
T(t) x_{n}=T(m \alpha+k \beta) x_{n}=T^{m}(\alpha)\left(T^{k}(\beta) x_{n}\right),
$$

which implies

$$
\left\|T(t) x_{n}-x_{n}\right\| \leq\left\|T^{m}(\alpha)\left(T^{k}(\beta) x_{n}\right)-T^{m}(\alpha) x_{n}\right\|+\left\|T^{m}(\alpha) x_{n}-x_{n}\right\|
$$

for any $n \geq 1$. Using Lemma 3.1 and the uniform continuity of $T^{m}(\alpha)$, we get $\lim _{n \rightarrow \infty}\left\|T(t) x_{n}-x_{n}\right\|=0$, i.e., $\left\{x_{n}\right\} \in \operatorname{AFPS}(T(t))$. Next assume that $t=m \alpha+k \beta$, where either $m$ or $k$ is negative. Without loss of generality assume $t=m \alpha-k \beta$, where $m, k \geq 0$. We have

$$
\left\|T(t) x_{n}-x_{n}\right\| \leq\left\|T(m \alpha-k \beta) x_{n}-T(m \alpha) x_{n}\right\|+\left\|T(m \alpha) x_{n}-x_{n}\right\| .
$$


Since $T(m \alpha)=T(m \alpha-k \beta) T(k \beta)$, and $T(m \alpha-k \beta)$ is uniformly continuous, Lemma 3.1 implies $\lim _{n \rightarrow \infty}\left\|T(t) x_{n}-x_{n}\right\|=0$, i.e., $\left\{x_{n}\right\} \in \operatorname{AFPS}(T(t))$. Hence

$$
\left\{x_{n}\right\} \in \bigcap_{t \in G_{+}(\alpha, \beta)} \operatorname{AFPS}(T(t)) .
$$

The following lemma, which can be found in any introductory course on real analysis, will be crucial to proving the first result on common approximate fixed point of semigroups.

Lemma 3.3 [17] Let $G$ be a nonempty additive subgroup of $\mathbb{R}$. Then $G$ is either dense in $\mathbb{R}$ or there exists $a>0$ such that $G=a \cdot \mathbb{Z}=\{a n, n \in \mathbb{Z}\}$. Therefore if $\alpha$ and $\beta$ are two real numbers such that $\frac{\alpha}{\beta}$ is irrational, then the set

$$
G(\alpha, \beta)=\{\alpha n+\beta m ; n, m \in \mathbb{Z}\}
$$

is dense in $\mathbb{R}$. In particular, the set $G_{+}(\alpha, \beta)=G(\alpha, \beta) \cap[0,+\infty)$ is dense in $[0,+\infty)$.

Theorem 3.1 Let $(X,\|\cdot\|)$ be a Banach space and $C \subset X$ be nonempty and bounded. Let $\mathcal{F}=\{T(t) ; t \geq 0\}$ be a one-parameter semigroup of uniformly continuous mappings from $C$ into $C$. Assume that $\mathcal{F}$ is strongly continuous. Let $\alpha$ and $\beta$ be two positive real numbers such that $\frac{\alpha}{\beta}$ is irrational. Then we have

$$
\operatorname{AFPS}(T(\alpha)) \cap \operatorname{AFPS}(T(\beta))=\operatorname{AFPS}(\mathcal{F}) .
$$

Proof Since $\operatorname{AFPS}(\mathcal{F}) \subset \operatorname{AFPS}(T(\alpha)) \cap \operatorname{AFPS}(T(\beta))$, it is enough to just prove $\operatorname{AFPS}(T(\alpha)) \cap \operatorname{AFPS}(T(\beta)) \subset \operatorname{AFPS}(\mathcal{F})$. Without loss of generality, assume that $\operatorname{AFPS}(T(\alpha)) \cap \operatorname{AFPS}(T(\beta))$ is not empty. Let $\left\{x_{n}\right\} \in \operatorname{AFPS}(T(\alpha)) \cap \operatorname{AFPS}(T(\beta))$. Lemma 3.2 implies that

$$
\left\{x_{n}\right\} \in \bigcap_{t \in G_{+}(\alpha, \beta)} \operatorname{AFPS}(T(t)) .
$$

From Lemma 3.3, we know that $G_{+}(\alpha, \beta)=G(\alpha, \beta) \cap[0,+\infty)$ is dense in $[0,+\infty)$. Let $t \in$ $[0,+\infty)$. Then there exists $t_{m} \in G_{+}(\alpha, \beta), m \geq 1$, such that $\lim _{m \rightarrow \infty} t_{m}=t$. We have

$$
\begin{aligned}
\left\|x_{n}-T(t) x_{n}\right\| & \leq\left\|x_{n}-T\left(t_{m}\right) x_{n}\right\|+\left\|T\left(t_{m}\right) x_{n}-T(t) x_{n}\right\| \\
& \leq\left\|x_{n}-T\left(t_{m}\right) x_{n}\right\|+\sup _{x \in C}\|T(t) x-T(t) x\| .
\end{aligned}
$$

Let $\varepsilon>0$. Since $\mathcal{F}$ is strongly continuous, there exists $m_{0} \geq 1$ such that for any $m \geq m_{0}$, we have

$$
\sup _{x \in C}\left\|T\left(t_{m}\right) x-T(t) x\right\|<\varepsilon
$$

Since $\left\{x_{n}\right\} \in \operatorname{AFPS}\left(T\left(m_{0}\right)\right)$ from Lemma 3.2, there exists $n_{0} \geq 1$ such that

$$
\left\|x_{n}-T\left(t_{m_{0}}\right)\left(x_{n}\right)\right\|<\varepsilon
$$


for any $n \geq n_{0}$. Hence

$$
\left\|x_{n}-T(t) x_{n}\right\| \leq\left\|x_{n}-T\left(t_{m_{0}}\right)\left(x_{n}\right)\right\|+\sup _{x \in C}\left\|T\left(t_{m_{0}}\right) x-T(t) x\right\|<2 \varepsilon
$$

for any $n \geq n_{0}$. Since $\varepsilon$ was arbitrarily positive, we conclude that $\lim _{n \rightarrow \infty}\left\|T(t) x_{n}-x_{n}\right\|=0$, i.e., $\left\{x_{n}\right\} \in \operatorname{AFPS}(T(t))$ for any $t \geq 0$.

As a corollary to Theorem 3.1, we get the following result.

Corollary 3.1 Let $(X,\|\cdot\|)$ be a Banach space and $C \subset X$ be nonempty and bounded. Let $\mathcal{F}=\{T(t) ; t \geq 0\}$ be a one-parameter semigroup of uniformly continuous mappings from $C$ into $C$. Assume that $\mathcal{F}$ is strongly continuous. Then we have

$$
\operatorname{AFPS}(\mathcal{F})=\operatorname{AFPS}(T(1)) \cap \operatorname{AFPS}(T(\pi))=\operatorname{AFPS}(T(1)) \cap \operatorname{AFPS}(T(\sqrt{2})) .
$$

All of the results obtained in this section may be easily stated in metric spaces. In the next section, we give an algorithm of how to construct an approximate fixed point sequence of two maps.

\section{Common approximate fixed points of two monotone mappings}

In this section we discuss a construction of a common approximate fixed point sequence of two monotone nonexpansive mappings defined on a Banach space $(X,\|\cdot\|)$ endowed with a partial order $\preceq$ as described before. Let $C \subset X$ be a nonempty convex subset. Let $J, H$ : $C \rightarrow C$ be two mappings. Fix $x_{1} \in C$, Das and Debata [18] studied the strong convergence of Ishikawa iterates $\left\{x_{n}\right\}$ defined by

$$
x_{n+1}=\alpha_{n} H\left(\beta_{n} J\left(x_{n}\right)+\left(1-\beta_{n}\right) x_{n}\right)+\left(1-\alpha_{n}\right) x_{n},
$$

where $\alpha_{n}, \beta_{n} \in[0,1]$. Under suitable assumptions, we will show that $\left\{x_{n}\right\}$ is an approximate fixed point sequence of both $J$ and $H$. Assume that $J$ and $H$ are monotone nonexpansive. Assume that there exists $x_{1} \in C$ such that $x_{1} \preceq J\left(x_{1}\right)$ and $x_{1} \preceq H\left(x_{1}\right)$. We will also assume that $H$ and $J$ have a common fixed point $p \in C$ such that $x_{1}$ and $p$ are comparable. Using the convexity properties of the partial order $\preceq$, we will easily show that $x_{n}$ and $p$ are comparable. Since $H$ and $J$ are monotone nonexpansive, we get $\left\|H\left(x_{n}\right)-p\right\| \leq\left\|x_{n}-p\right\|$ and $\left\|J\left(x_{n}\right)-p\right\| \leq\left\|x_{n}-p\right\|$ for any $n \geq 1$. Hence

$$
\begin{aligned}
\left\|x_{n+1}-p\right\| & =\left\|\alpha_{n} H\left(y_{n}\right)+\left(1-\alpha_{n}\right) x_{n}-p\right\| \\
& \leq \alpha_{n}\left\|H\left(y_{n}\right)-p\right\|+\left(1-\alpha_{n}\right)\left\|x_{n}-p\right\| \\
& \leq \alpha_{n}\left\|y_{n}-p\right\|+\left(1-\alpha_{n}\right)\left\|x_{n}-p\right\| \\
& =\alpha_{n}\left\|\beta_{n} J\left(x_{n}\right)+\left(1-\beta_{n}\right) x_{n}-p\right\|+\left(1-\alpha_{n}\right)\left\|x_{n}-p\right\| \\
& \leq \alpha_{n}\left[\beta_{n}\left\|J\left(x_{n}\right)-p\right\|+\left(1-\beta_{n}\right)\left\|x_{n}-p\right\|\right]+\left(1-\alpha_{n}\right)\left\|x_{n}-p\right\| \\
& \leq \alpha_{n}\left[\beta_{n}\left\|x_{n}-p\right\|+\left(1-\beta_{n}\right)\left\|x_{n}-p\right\|\right]+\left(1-\alpha_{n}\right)\left\|x_{n}-p\right\| \\
& \leq\left\|x_{n}-p\right\|,
\end{aligned}
$$


where $y_{n}=\beta_{n} J\left(x_{n}\right)+\left(1-\beta_{n}\right) x_{n}$ for any $n \geq 1$. This proves that $\left\{\left\|x_{n}-p\right\|\right\}$ is decreasing, which implies that $\lim _{n \rightarrow \infty}\left\|x_{n}-p\right\|$ exists. Using the above inequalities, we get

$$
\begin{aligned}
\lim _{n \rightarrow \infty}\left\|x_{n}-p\right\| & =\lim _{n \rightarrow \infty}\left\|\alpha_{n} H\left(y_{n}\right)+\left(1-\alpha_{n}\right) x_{n}-p\right\| \\
& =\lim _{n \rightarrow \infty}\left[\alpha_{n}\left\|H\left(y_{n}\right)-p\right\|+\left(1-\alpha_{n}\right)\left\|x_{n}-p\right\|\right] \\
& =\lim _{n \rightarrow \infty}\left[\alpha_{n}\left\|y_{n}-p\right\|+\left(1-\alpha_{n}\right)\left\|x_{n}-p\right\|\right] \\
& =\lim _{n \rightarrow \infty}\left[\alpha_{n}\left\|\beta_{n} J\left(x_{n}\right)+\left(1-\beta_{n}\right) x_{n}-p\right\|+\left(1-\alpha_{n}\right)\left\|x_{n}-p\right\|\right] \\
& =\lim _{n \rightarrow \infty}\left[\alpha_{n}\left(\beta_{n}\left\|J\left(x_{n}\right)-p\right\|+\left(1-\beta_{n}\right)\left\|x_{n}-p\right\|\right)+\left(1-\alpha_{n}\right)\left\|x_{n}-p\right\|\right] .
\end{aligned}
$$

Before we state the main theorem of this section, let us recall the definition of a uniformly convex Banach space.

Definition 4.1 [19] Let $(X,\|\cdot\|)$ be a Banach space. $X$ is said to be uniformly convex if and only if for any $\varepsilon>0$, we have $\delta_{X}(\varepsilon)>0$, where

$$
\delta_{X}(\varepsilon)=\inf \left\{1-\frac{1}{2}\|x+y\| ;\|x\| \leq 1,\|y\| \leq 1 \text { and }\|x-y\| \geq \varepsilon\right\} .
$$

It is well known that if $X$ is uniformly convex, then $X$ is reflexive [19]. Moreover, we have [20]

$$
\|\alpha x+(1-\alpha) y\| \leq 1-\delta_{X}(2 \min \{\alpha, 1-\alpha\} \varepsilon)
$$

for any $\alpha \in(0,1), \varepsilon>0$ and $x, y \in X$ such that $\|x\| \leq 1,\|y\| \leq 1$ and $\|x-y\| \geq \varepsilon$.

The following lemma will be needed to prove the main result of this section.

Lemma 4.1 [20] Let $(X,\|\cdot\|)$ be a uniformly convex Banach space. Let $\left\{x_{n}\right\}$ and $\left\{y_{n}\right\}$ be in $X$ such that $\limsup _{n \rightarrow \infty}\left\|x_{n}\right\| \leq R, \lim \sup _{n \rightarrow \infty}\left\|y_{n}\right\| \leq R$, and $\lim _{n \rightarrow \infty}\left\|\alpha_{n} x_{n}+\left(1-\alpha_{n}\right) y_{n}\right\|=R$, where $\alpha_{n} \in[a, b]$, with $0<a \leq b<1$, and $R \geq 0$. Then we have

$$
\lim _{n \rightarrow \infty}\left\|x_{n}-y_{n}\right\|=0
$$

Next we state the main result of this section.

Theorem 4.1 Let $C$ be a nonempty, closed and convex subset of a uniformly convex Banach space $(X,\|\cdot\|)$. Let $H, J: C \rightarrow C$ be monotone nonexpansive mappings such that $H$ is uniformly continuous. Assume that there exists $x_{1} \in C$ such that $x_{1} \preceq J\left(x_{1}\right)$ and $x_{1} \preceq H\left(x_{1}\right)$. We will also assume that $H$ and $J$ have a common fixed point $p \in C$ such that $x_{1}$ and $p$ are comparable. Consider the sequence $\left\{x_{n}\right\}$ defined by $x_{1}$ and the recurrent formula (DD). Assume that $\alpha_{n}, \beta_{n} \in[\alpha, \beta]$, with $0<\alpha \leq \beta<1$, then

$$
\lim _{n \rightarrow \infty}\left\|x_{n}-H\left(x_{n}\right)\right\|=0 \text { and } \lim _{n \rightarrow \infty}\left\|x_{n}-J\left(x_{n}\right)\right\|=0
$$

i.e., $\left\{x_{n}\right\} \in \operatorname{AFPS}(J) \cap \operatorname{AFPS}(H)$. 
Proof We have already seen that $\left\{\left\|x_{n}-p\right\|\right\}$ is decreasing. Set $c=\lim _{n \rightarrow \infty}\left\|x_{n}-p\right\|$. If $c=0$, then all the conclusions are trivial. Therefore we will assume that $c>0$. We have already seen that

$$
\begin{aligned}
\lim _{n \rightarrow \infty}\left\|x_{n}-p\right\| & =\lim _{n \rightarrow \infty}\left\|\alpha_{n} H\left(y_{n}\right)+\left(1-\alpha_{n}\right) x_{n}-p\right\| \\
& =\lim _{n \rightarrow \infty}\left[\alpha_{n}\left\|H\left(y_{n}\right)-p\right\|+\left(1-\alpha_{n}\right)\left\|x_{n}-p\right\|\right] .
\end{aligned}
$$

We claim that $\lim _{n \rightarrow \infty}\left\|H\left(y_{n}\right)-p\right\|=c$. Indeed, let $\mathcal{U}$ be a nontrivial ultrafilter over $\mathbb{N}$. Then we have $\lim _{n, \mathcal{U}} \alpha_{n}=\alpha_{\infty} \in[\alpha, \beta]$ and $\lim _{n, \mathcal{U}}\left\|x_{n}-p\right\|=c$. Hence

$$
c=\alpha_{\infty} \lim _{n, \mathcal{U}}\left\|H\left(y_{n}\right)-p\right\|+\left(1-\alpha_{\infty}\right) c
$$

Since $\alpha_{\infty} \neq 0$, we get $\lim _{n, \mathcal{U}}\left\|H\left(y_{n}\right)-p\right\|=c$. Since $\mathcal{U}$ was arbitrary, we get $\lim _{n \rightarrow \infty} \| H\left(y_{n}\right)-$ $p \|=c$ as claimed. Therefore we have

$$
\lim _{n \rightarrow \infty}\left\|x_{n}-p\right\|=\lim _{n \rightarrow \infty}\left\|H\left(y_{n}\right)-p\right\|=\lim _{n \rightarrow \infty}\left\|\alpha_{n} H\left(y_{n}\right)+\left(1-\alpha_{n}\right) x_{n}-p\right\| .
$$

Using Lemma 4.1, we get $\lim _{n \rightarrow \infty}\left\|H\left(y_{n}\right)-x_{n}\right\|=0$. Since we already proved

$$
\lim _{n \rightarrow \infty}\left\|x_{n}-p\right\|=\lim _{n \rightarrow \infty}\left[\alpha_{n}\left\|y_{n}-p\right\|+\left(1-\alpha_{n}\right)\left\|x_{n}-p\right\|\right]
$$

a similar argument will show that $\lim _{n \rightarrow \infty}\left\|y_{n}-p\right\|=\lim _{n \rightarrow \infty}\left\|x_{n}-p\right\|$. Moreover, we have

$$
\lim _{n \rightarrow \infty}\left\|x_{n}-p\right\|=\lim _{n \rightarrow \infty}\left[\alpha_{n}\left(\beta_{n}\left\|J\left(x_{n}\right)-p\right\|+\left(1-\beta_{n}\right)\left\|x_{n}-p\right\|\right)+\left(1-\alpha_{n}\right)\left\|x_{n}-p\right\|\right] .
$$

If we use again ultrafilters, one will easily prove that $\lim _{n \rightarrow \infty}\left\|J\left(x_{n}\right)-p\right\|=\lim _{n \rightarrow \infty}\left\|x_{n}-p\right\|$. Hence we have

$$
\lim _{n \rightarrow \infty}\left\|x_{n}-p\right\|=\lim _{n \rightarrow \infty}\left\|J\left(x_{n}\right)-p\right\|=\lim _{n \rightarrow \infty}\left\|\beta_{n} J\left(x_{n}\right)+\left(1-\beta_{n}\right) x_{n}-p\right\| .
$$

Using again Lemma 4.1 , we get $\lim _{n \rightarrow \infty}\left\|J\left(x_{n}\right)-x_{n}\right\|=0$. Since

$$
\lim _{n \rightarrow \infty}\left\|y_{n}-x_{n}\right\|=\lim _{n \rightarrow \infty} \beta_{n}\left\|J\left(x_{n}\right)-x_{n}\right\|=0,
$$

and $H$ is uniformly continuous, we get $\lim _{n \rightarrow \infty}\left\|H\left(x_{n}\right)-H\left(y_{n}\right)\right\|=0$. Combined with $\lim _{n \rightarrow \infty}\left\|H\left(y_{n}\right)-x_{n}\right\|=0$, we get

$$
\lim _{n \rightarrow \infty}\left\|H\left(x_{n}\right)-x_{n}\right\|=0
$$

Remark 4.1 If we assume that $\alpha_{n}=\alpha$ and $\beta_{n}=\beta$ with $\alpha, \beta \in(0,1)$, then under the assumptions of Theorem 4.1, we can prove that

$$
x_{n} \leq x_{n+1} \quad \text { and } \quad y_{n} \leq y_{n+1}
$$

for any $n \geq 1$. Since $\left\{\left\|x_{n}-p\right\|\right\}$ and $\left\{\left\|y_{n}-p\right\|\right\}$ are convergent, then both $\left\{x_{n}\right\}$ and $\left\{y_{n}\right\}$ are bounded. Therefore, they have a weak-cluster point since $X$ is reflexive. Using the 
convexity properties of the partial order $\preceq$ in $X$, we will conclude that in fact $\left\{x_{n}\right\}$ and $\left\{y_{n}\right\}$ are weakly convergent. It is not clear to us that the weak-limit of $\left\{x_{n}\right\}$ is a fixed point of $H$ and $J$. This will be the case if we have a demi-closed property for monotone nonexpansive mappings. Otherwise, we may assume that $X$ satisfies the Opial condition [21]. In this case the weak-limit of $\left\{x_{n}\right\}$ will be a fixed point of $T$ and $S$. As an example of a Banach space which satisfies all of the above assumptions, one may take $X=\ell^{p}, 1<p<+\infty$.

\section{Competing interests}

The authors declare that they have no competing interests.

Authors' contributions

All authors contributed equally to the writing of this paper. All authors read and approved the final manuscript.

\section{Author details}

${ }^{1}$ Department of Mathematics, College of Sciences, King Saud University, Riyadh, Saudi Arabia. ${ }^{2}$ Department of Mathematical Sciences, The University of Texas at El Paso, El Paso, TX 79968, USA. ${ }^{3}$ Department of Mathematics and Statistics, King Fahd University of Petroleum \& Minerals, Dhahran, 31261, Saudi Arabia.

\section{Acknowledgements}

The authors would like to extend their sincere appreciation to the Deanship of Scientific Research at King Saud University for funding this Research group No. RG-1435-079.

Received: 4 June 2015 Accepted: 13 August 2015 Published online: 04 September 2015

\section{References}

1. Belluce, LP, Kirk, WA: Fixed-point theorems for families of contraction mappings. Pac. J. Math. 18, $213-217$ (1966)

2. Belluce, LP, Kirk, WA: Nonexpansive mappings and fixed-points in Banach spaces. III. J. Math. 11, 474-479 (1967)

3. Browder, FE: Nonexpansive nonlinear operators in a Banach space. Proc. Natl. Acad. Sci. USA 54, 1041-1044 (1965)

4. Bruck, RE: Properties of fixed point sets of non-expansive mappings in Banach spaces. Trans. Am. Math. Soc. 179, 251-262 (1973)

5. Lim, TC: A fixed point theorem for families of nonexpansive mappings. Pac. J. Math. 53, 487-493 (1974)

6. Ran, ACM, Reurings, MCB: A fixed point theorem in partially ordered sets and some applications to matrix equations. Proc. Am. Math. Soc. 132, 1435-1443 (2004)

7. Turinici, M: Abstract comparison principles and multivariable Gronwall-Bellman inequalities. J. Math. Anal. Appl. 117 , 100-127 (1986)

8. Turinici, M: Fixed points for monotone iteratively local contractions. Demonstr. Math. 19, 171-180 (1986)

9. Nieto, JJ, Rodríguez-López, R: Contractive mapping theorems in partially ordered sets and applications to ordinary differential equations. Order 22, 223-239 (2005)

10. Jachymski, J: The contraction principle for mappings on a metric space with a graph. Proc. Am. Math. Soc. 136, 1359-1373 (2007)

11. Turinici, M: Ran and Reurings theorems in ordered metric spaces. J. Indian Math. Soc. 78, 207-2014 (2011)

12. Goebel, K, Kirk, WA: Topics in Metric Fixed Point Theory. Cambridge Stud. Adv. Math., vol. 28. Cambridge University Press, Cambridge (1990)

13. Khamsi, MA, Kirk, WA: An Introduction to Metric Spaces and Fixed Point Theory. Wiley, New York (2001)

14. Pavel, NH: Nonlinear Evolution Operators and Semigroups: Applications to Partial Differential Equations. Lecture Notes in Mathematics. Springer, Berlin (1987)

15. Pazy, A: Semigroups of Linear Operators and Applications to Partial Differential Equations. Applied Mathematical Sciences, vol. 44. Springer, New York (1983)

16. Reich, S, Shoikhet, D: Nonlinear Semigroups, Fixed Points, and Geometry of Domains in Banach Spaces. Imperial College Press, London (2005)

17. Stewart, I, Tall, D: Algebraic Number Theory and Fermat's Last Theorem, 3rd edn. AK Peters, Wellesley (2001)

18. Das, G, Debata, P: Fixed points of quasi-nonexpansive mappings. Indian J. Pure Appl. Math. 17, 1263-1269 (1986)

19. Beauzamy, B: Introduction to Banach Spaces and Their Geometry. Notas de Mathematica, vol. 68. North-Holland, Amsterdam (1982)

20. Fukhar-ud-din, H, Khamsi, MA: Approximating common fixed points in hyperbolic spaces. Fixed Point Theory Appl. 2014, 113 (2014). doi:10.1186/1687-1812-2014-113

21. Opial, Z: Weak convergence of the sequence of successive approximations for nonexpansive mappings. Bull. Am Math. Soc. 73, 591-597 (1967) 